Remote Sensing Of The Oceans And Inland Waters: Techniques, Applications, And Challenges

2016, Volume 9878T Pages 1-20

http://dx.doi.org/10.1117/12.2225619

http://archimer.ifremer.fr/doc/00363/47468/

(C) (2016) COPYRIGHT Society of Photo-Optical Instrumentation

Engineers (SPIE).

\title{
CFOSAT: A new Chinese-French satellite for joint observations of ocean wind vector and directional spectra of ocean waves
}

\author{
Hauser D. ${ }^{1,{ }^{*}}$, Tison C. ${ }^{2}$, Amiot T. ${ }^{2}$, Delaye L. ${ }^{1}$, Mouche Alexis ${ }^{3}$, Guitton Gilles ${ }^{3}$, Aouf L. ${ }^{4}$, \\ Castillan P. ${ }^{2}$
}

${ }^{1}$ CNRS, LATMOS, UVSQ, UPMC, 11 bd dAlembert, F-78280 Guyancourt, France.

${ }^{2}$ BCNES, Toulouse, France.

${ }^{3}$ LOS, IFREMER, Plouzane, France.

${ }^{4}$ Meteo France, Toulouse, France.

* Corresponding author : D. Hauser, email address : danielc.hauser@latmos.ispl.fr

\begin{abstract}
:
CFOSAT (the China France Oceanography Satellite) is a joint mission from the Chinese and French Space Agencies, devoted to the observation ocean surface wind and waves so as to improve wind and wave forecast for marine meteorology, ocean dynamics modeling and prediction, climate variability knowledge, fundamental knowledge of surface processes. Currently under Phase D (manufacturing phase), the launch is now planned for mid-2018 the later. The CFOSAT will carry two payloads, both Ku-Band radar: the wave scatterometer (SWIM) and the wind scatterometer (SCAT). Both instruments are based on new concepts with respect to existing satellite-borne wind and wave sensors. Indeed, one of the originalities of CFOSAT is that it will provide simultaneously and in the same zone, the directional spectra of ocean waves and the wind vector. The concept used to measure the directional spectra of ocean waves has never been used from space until now: it is based on a near-nadir incidence pointing, rotating fan-beam radar, used in a real-aperture mode. In this paper we present the CFOSAT mission, its objectives and main characteristics. We then focus on the SWIM instrument, the expected geophysical products and performances. Finally, we present ongoing studies based on existing satellite data of directional spectra of ocean waves (Sentinel-1,..) and carried out in preparation to CAL/VAL activities and to future data exploitation.
\end{abstract}

Keywords : satellite, radar, surface ocean waves, surface ocean wind, scatterometer, CFOSAT 


\section{INTRODUCTION}

The Chinese and French Space Agencies are jointly preparing an innovative mission, CFOSAT (China France Oceanography Satellite) devoted to the monitoring of the ocean surface and its related science and applications. For the very first time, both wind and wave vectors will be measured at the global ocean surface. This will be achieved thanks to the two-instruments, both of them being innovative instruments in terms of geometry and design:

- SWIM (Surface Wave Investigation and Monitoring) a near-nadir (0 to $10^{\circ}$ incidence) real-aperture Ku-Band azimuthally scanning radar designed for measuring the directional spectra of ocean waves according ;

- SCAT a wind scatterometer SCAT to measure the wind vector, radar in Ku-Band aiming at moderate incidence angles $\left(26^{\circ}\right.$ to $\left.46^{\circ}\right)$ with a rotating fan-beam antenna (see [1])

With respect to existing satellite missions, the originality of CFOSAT is that it will provide at the global scale, colocated wind vector fields and directional spectra of ocean waves for wavelengths larger than about $70 \mathrm{~m}$. It will also provide normalized radar cross-section in multi-incidence and multi-azimuth geometry which can be used on one hand to improve the inversion algorithms for estimating wind speed and significant wave height and on the other hand to characterize the small scale roughness of all types of surfaces. CFOSAT will contribute to the global wind field observations in complement to existing scatterometer missions (e.g. ASCAT on METOP, SCAT on HY-2A), and to

* daniele.hauser@latmos.ipsl.fr 
provisioning of wind speed and significant wave height in complement to other altimeter missions (like Jason or HY-2 series, Sentinel-3). Furthermore, it will provide new information on wave properties with respect to SAR missions (like Sentinel-1), by giving access to directional spectra of ocean waves not only for the long swells but also for wind waves and mixed sea conditions whatever is the direction of these waves. Indeed, the SWIM instrument is based on the realaperture rotating concept proposed by [2], specified by [3] for satellite implementation, and implemented on airplanes since the 90's $[2,4,5]$. This concept avoids the limitations induced by the imaging mechanism occurring with SAR data over the moving ocean surface [6].

Here, we present the general scientific objectives and main characteristics of the mission, the expected geophysical products and their performance, and we present some ongoing studies carried out in preparation of this mission on timespace analysis of wave fields and on data assimilation in numerical wave forecasting systems.

\section{SCIENTIFIC OBJECTIVES}

CFOSAT will provide global observations of surface wind and spectral characteristics of the surface ocean waves. The objective is multifold. It will serve both operational needs for the meteorological and wave forecast, and research needs to improve our knowledge on the hydrodynamics of the waves, on the interactions between waves and the atmospheric or oceanic layers close to the surface. Furthermore it will be a useful tool to better characterize the backscattering of ocean and land in a diversity of geometrical configuration, providing new information on the roughness characteristics and associated surface characterization (sea ice and ice sheet, vegetation and arid zones).

Improving the numerical modeling and prediction of ocean surface wind and waves remains a crucial need for many applications over the ocean (offshore operations and engineering, fisheries, ship routing, coastal or harbor management. etc). The performance of wave models has significantly improved in the last decade [7] thanks to improved accuracy in the forcing wind fields, as well as to improved parameterization of wave processes, and to the assimilation of significant wave heights from altimeters. However improvements are still needed, in particular for high sea-state conditions, extreme events (hurricanes, typhoons), rapidly evolving storms, and for providing accurate prediction not only in terms of wave height but also in terms of peak wavelength and direction. The accurate prediction of low frequency swell, propagating great distances from the generation regions, remains a priority for many applications.

It is known that wind forces the wave generation and that in turn surface waves modify the atmospheric surface layer and hence the air/sea exchanges both within and outside of the generation area [8]. For this reason, more and more often, atmospheric and wave models are coupled within forecast systems. Recent studies coupling wave and oceanic models [9] also show that the impact of waves on ocean surface layers greatly depends on type of sea-state (growing or dissipating stage). Hence, CFOSAT will provide original data to take wave effects into account as it will provide co-located and simultaneous measurements of surface wind and waves at scales compatible with global numerical models. This will on one hand allow to better constrain the models by the wind and wave observations through assimilation and on the other hand it will help to better quantify and parameterize the effect of waves on the atmosphere and ocean (impact on heat and momentum exchanges, impact on sea ice, impact on kinetic energy flux to the ocean,...). It will also contribute to better characterize the wind and waves in extreme events like tropical or mid latitude storms.

Better knowledge of wind and wave climatology in the context of global climate change becomes an increasing subject of interest [10]. Although altimeter missions already provide multi-year statistics of significant wave height, and scatterometer statistics on wind speed, there is a lack of information on wave system components and their dominant frequency and direction. CFOSAT will contribute to establish a global climatology with joint statistics on wind, significant wave height, dominant wavelengths and directions. It will also be possible to relate wave climatology at certain locations to climatology of the generating mid-latitude or tropical storms, whose positions, intensity or number might be affected by climate change.

CFOSAT will also be a unique tool to study physical processes such as the interaction of long swell with wind waves and turbulence generated locally, energy focusing related to surface current or crossed seas, potentially associated with dangerous sea state. This will also permit to characterize the swell propagation and its dissipation over long distances under various wind and surface current conditions. Furthermore, thanks to its multiple incidence configuration CFOSAT will provide information on short ripples on the surface (of the order of a few centimeters), intermediate waves (few tens 
of centimeters to a few meters and long waves (several tens to several hundred of meters). Exchanges of energy take place between these different ranges of waves, which also depend on external conditions such as surface current. So there will be a unique opportunity to study the time and space evolution of the waves at different scales, taking into account their propagation direction. Thanks to its multi-incidence configuration which will provide simultaneous information on short waves (Bragg scattering from SCAT at medium incidence), mean square slope (from low incidence angle observations), and long waves, CFOSAT will be a strong opportunity to progress in the understanding of the relations between wind, wind stress, wind-generated waves, and long waves.

Mean momentum from the atmosphere is transferred to the ocean in two ways, i.e. as direct surface stress-driven Ekman currents, and as mean drift associated with the wave motion. Processes like surface winds, viscous dissipation and wave breaking will alter the wave amplitude in time and space, converting mean momentum from waves to ocean currents. Hence measuring simultaneously wind and wave fields including the directional properties of waves, will help to better understand the transfer of momentum from atmosphere to ocean and take it into account in ocean modeling.

Finally multi-angular (incidence and azimuth) Ku-Band backscattering from SWIM and SCAT over land, sea ice and polar ice-sheet will provide new information to characterize these types of surface and their seasonal or multi-year evolution.

\section{CFOSAT MAIN CHARACTERISTICS}

CFOSAT is a common initiative of the Chinese and French Space Agencies (resp. CNSA and CNES) started in 2006.

The system consists of a LEO polar sun-synchronous orbit system with local time ascending pass at the equator at 7:00 am. The orbit altitude will be about $519 \mathrm{~km}$ altitude at the equator. Data will be transmitted to Mission Centers using several receiving stations. Thanks to a set of two polar stations, the system will have the capability to achieve near-real time transmission (i.e. less than 3 hours after the acquisition) of the global set of observations in order to feed operational atmospheric or wave prediction systems for assimilation and forecast processes.

China provides the satellite platform (a "CAST 2000" platform), the satellite launch (a CZ-2C rocket), the satellite control, and the wind Ku-Band scatterometer (SCAT). France provides the wave Ku-Band scatterometer (SWIM). Both countries contribute to the ground segment. The Chinese contribution to the Ground Segment is composed of a "Satellite Control Center" located in Xian (China), several telemetry, tracking and command ground stations, 3 X-band receiving stations located in China, and a Mission Center for data processing, distribution and archiving. The French CFOSAT Ground Segment is composed of 2 X-band Stations, located in Kiruna (Sweden) and Inuvik (Canada), and two mission centers: one operated by CNES in Toulouse (France) for near-real time processing distribution of data, and data archiving, and a second one for differed-time data processing, distribution and archiving operated by Ifremer in Brest (France). From the CNES Center, the near-real time wind and wave products shall be made available at final users in operational centers within 3 hours from acquisition time, with an availability of $75 \%$ - goal $85 \%$ and in differed time to the scientific community. This scheme is completed in France by expert groups for wind and wave products and by instrumental expert group.

Feasibility and preliminary design phases (A/B phases) were successfully achieved from 2006 until 2010. The project started the detailed design phase $\mathrm{C}$ in 2011. The manufacturing phase $\mathrm{D}$ is now on going and will be followed by satellite integration and tests (in 2017). The launch and the assessment phase completion should lead to deliver a fully validated system in orbit in 2018. The mission lifetime is at least 3 years.

As mentioned above, the platform will embark two payloads (see artists view in Fig.1); both are Ku-band radar scanning around the vertical axis:

- the wave spectrometer SWIM $(13.575 \mathrm{GHz})$, provided by CNES and manufactured by Thales Alenia Space, is a rotating 6-beams radar at small incidence $\left(0^{\circ}\right.$ to $\left.10^{\circ}\right)$,

- the wind scatterometer SCAT $(13.256 \mathrm{GHz})$ designed by MiRS Lab of National Space Science Center, Chinese Academy of Sciences, and provided by CNSA is a rotating fan-beam radar pointing at medium incidence angles $\left(26^{\circ}\right.$ to $\left.46^{\circ}\right)$ with a dual polarization antenna system.

With an orbital repetition cycle of 13 days and accounting for the instrument geometry the system will provide a global coverage within 3 days for wind fields (SCAT) and almost global for waves (SWIM). 


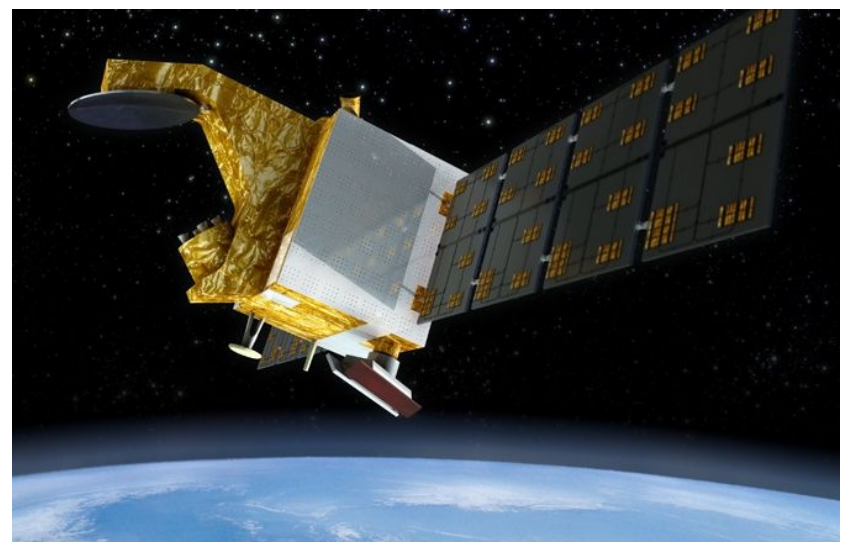

Figure 1. Artist view of the CFOSAT satellite. The SWIM antenna system is on the left. It is composed of a rotating plate with 6 feed-horns and an offset parabolic antenna .The SCAT antenna system is on the bottom right. It is a set of two opposed plane antenna. Other antennas on Earth face are for TM/TC (X and S-bands).. (C CNES/ Gekko.

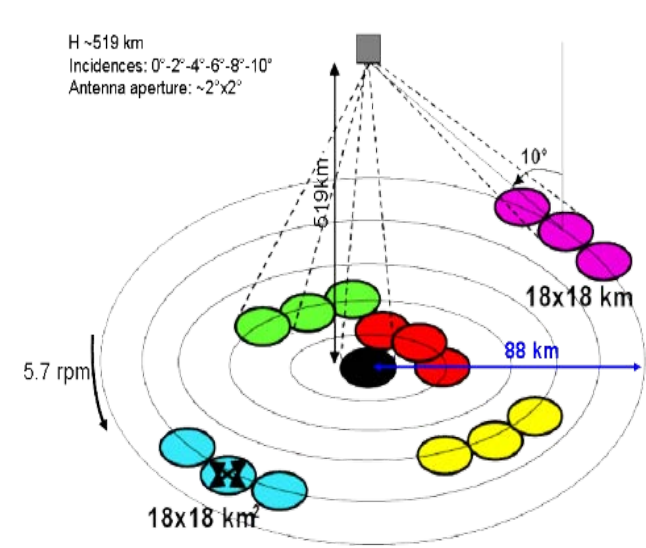

Figure 2: SWIM geometry with its six rotating incidence beams from 0 to $10^{\circ}$. The footprint dimension of each beam is about $18 \mathrm{~km} \times 18 \mathrm{~km}$. The diameter of the full swath is about $180 \mathrm{~km}$

\section{SCIENTIFIC REQUIREMENTS}

The main scientific requirements on the data are the following:

Surface ocean waves (from SWIM):

- Wave directional spectra at a scale of 70 x $90 \mathrm{~km}$ on each side of the track for wavelength range $[70 \mathrm{~m}-500 \mathrm{~m}]$ retrieved with a $10 \%$ accuracy on wavelength, $15^{\circ}$ accuracy on direction (with however a $180^{\circ}$ ambiguity in direction), about 15 to $20 \%$ accuracy in energy in the energy containing part of the spectrum

- Significant wave height along track (SWIM) with a $10 \%$ (or $50 \mathrm{~cm}$ whichever is better) accuracy

Surface wind:

- wind vector over swath of $\pm \sim 600 \mathrm{~km}$ across-track (SCAT) within $50 \mathrm{~km}$ resolution cells $(25 \mathrm{~km}$ experimental) and an accuracy of $2 \mathrm{~m} / \mathrm{s}$ or $10 \% \mathrm{rms}$ in the range $4 \sim 24 \mathrm{~m} / \mathrm{s}$, and a $20^{\circ}$ accuracy in direction

- wind speed along track (from SWIM nadir beam) with an accuracy of $2 \mathrm{~m} / \mathrm{s} \mathrm{rms}$

Normalized radar backscattering cross-sections $\sigma_{0}$ :

- mean profiles of $\sigma_{0}$ as a function of incidence angle $\left(0-10^{\circ}\right)$ and azimuth angle $\left(0-180^{\circ}\right)$ from SWIM, at a scale of $70 \times 90 \mathrm{~km}$ on each side of the track, with a $\pm 1 \mathrm{~dB}$ absolute accuracy, and \pm 0.1 to $0.2 \mathrm{~dB}$ relative accuracy

- mean values of $\sigma_{0}$ within $50 \mathrm{~km}$ resolution cells ( $25 \mathrm{~km}$ experimental) from SCAT, with a $\pm 1 \mathrm{~dB}$ absolute accuracy, and \pm 0.1 to $0.2 \mathrm{~dB}$ relative accuracy

\section{THE SWIM INSTRUMENT}

SWIM is a Ku-band real aperture radar following the concepts developed in [2, 3]. It illuminates the surface sequentially with 6 incidence angles: $0^{\circ}, 2^{\circ}, 4^{\circ}, 6^{\circ}, 8^{\circ}$ and $10^{\circ}$ (Figure 2). In order to acquire data in all azimuth orientations, the antenna is rotating at a speed rate of $5.6 \mathrm{rpm}$. The six beams enable to measure several geophysical parameters:

- all beams: backscattering coefficient profiles from $0^{\circ}$ to $10^{\circ}$ of each surface, 
- $\quad$ nadir beam $\left(0^{\circ}\right)$ : SWH and surface wind over sea surfaces, similarly as nadir altimeter,

- $\quad 6^{\circ}, 8^{\circ}$ and $10^{\circ}$ beams (named "spectrum" beams): 2D surface ocean wave spectra.

Table 1 summarizes the main characteristics of the instrument. Table 2 provides the parameters of the six incidence beams of SWIM. Note that some integration time-duration and PRF have been recently changed with respect to what was presented in previous communications, in order to guarantee compatibility with electrical constraints of the platform.

Table 1. SWIM main parameters

\begin{tabular}{|l|c|}
\hline Frequency & $13.575 \mathrm{GHz}$ \\
\hline Useful bandwidth & $320 \mathrm{MHz}$ \\
\hline Useful pulse duration & $50 \mu \mathrm{s}$ \\
\hline Peak power & $120 \mathrm{~W}$ \\
\hline Incidence angles (on ground) & $0^{\circ}-2.43^{\circ}-4^{\circ}-6^{\circ}-8^{\circ}-10^{\circ}$ \\
\hline PRF & from 5 to $5.4 \mathrm{kHz}$ \\
\hline Antenna rotation speed & $5.6 \mathrm{rpm}$ \\
\hline Antenna diameter & $90 \mathrm{~cm}$ \\
\hline Antenna 3dB aperture $\left(0^{\circ}\right.$ and $\left.2^{\circ}\right)$ & $>1.5^{\circ}$ \\
\hline Antenna 3dB aperture (from $4^{\circ}$ to $\left.10^{\circ}\right)$ & $>1.7^{\circ}$ \\
\hline Polarization & Linear (VV in rotation) \\
\hline
\end{tabular}

Table 2. Incidence beams parameter for each incidence beam $\left(0^{\circ}\right.$ to $10^{\circ}$ ). $\mathrm{N}_{\text {IMP }}$ is the number of on-board integrated samples (over each cycle), RA the ambiguity rank, PRF the minimum and maximum pulse repetition frequency along the orbit (according to local altitude), $\mathrm{T}_{\text {cycle }}$ is the corresponding minimum and maximum cycle duration, the last line is the cycle duration without accounting for rank ambiguity.

\begin{tabular}{|c|c|c|c|c|c|c|}
\hline & $0^{\circ}$ & $2^{\circ}$ & $4^{\circ}$ & $6^{\circ}$ & $8^{\circ}$ & $10^{\circ}$ \\
\hline NIMP & 264 & 97 & 97 & 156 & 186 & 204 \\
\hline $\mathrm{RA}$ & 18 & 18 & 18 & 18 & 18 & 18 \\
\hline $\mathrm{PRF}_{\min }$ & $5093 \mathrm{~Hz}$ & $5079 \mathrm{~Hz}$ & $5079 \mathrm{~Hz}$ & $5065 \mathrm{~Hz}$ & $5037 \mathrm{~Hz}$ & $5023 \mathrm{~Hz}$ \\
\hline $\mathrm{PRF}_{\max }$ & $5427 \mathrm{~Hz}$ & $5427 \mathrm{~Hz}$ & $5411 \mathrm{~Hz}$ & $5395 \mathrm{~Hz}$ & $5379 \mathrm{~Hz}$ & $5348 \mathrm{~Hz}$ \\
\hline $\mathrm{T}_{\text {crde min }}$ & $52.0 \mathrm{~ms}$ & $21.3 \mathrm{~ms}$ & $21.3 \mathrm{~ms}$ & $32.3 \mathrm{~ms}$ & $37.9 \mathrm{~ms}$ & $41.5 \mathrm{~ms}$ \\
\hline$T_{\text {crrde max }}$ & $55.4 \mathrm{~ms}$ & $22.6 \mathrm{~ms}$ & $22.6 \mathrm{~ms}$ & $34.4 \mathrm{~ms}$ & $40.4 \mathrm{~ms}$ & $44.2 \mathrm{~ms}$ \\
\hline $\begin{array}{c}T_{\text {crve max }} \\
\text { (hors RAxPRI) }\end{array}$ & $51.8 \mathrm{~ms}$ & $19.0 \mathrm{~ms}$ & $19.1 \mathrm{~ms}$ & $30.8 \mathrm{~ms}$ & $36.8 \mathrm{~ms}$ & $40.6 \mathrm{~ms}$ \\
\hline
\end{tabular}

The cycle is the consecutive time spent at a given incidence, and the macro-cycle is the elementary repeated set of cycles, which combines all incidences. The nominal macro-cycle is $\left\{0^{\circ}, 2^{\circ}, 4^{\circ}, 6^{\circ}, 8^{\circ}, 10^{\circ}\right\}$ but it can be remotely modified during flight. Note that the PRF is adaptive along the orbit (according to local altitude) whereas the number of averaged pulses per cycle is kept constant (see Table 2).

The main modes of the instrument functioning are (Fig. 3):

- STAND-BY when SWIM is ready and waits for the transition to one of the measurement modes,

- ACQUISITION when SWIM computes the acquisition time windows from the detection of the nadir echo (temporary mode before switching to tracking),

- TRACKING when SWIM measures the backscattered power of the echo for successive cycles and macrocycles; this is the scientific mode,

- CAL1 and CAL2 when SWIM performs internal calibration (including rotary part of the antenna calibration), or thermal noise acquisition.

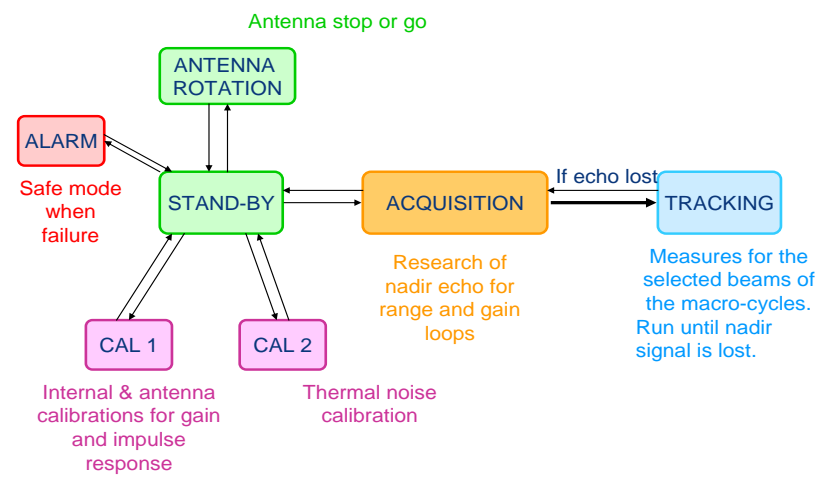

Figure 3. Main modes of SWIM 
It is also possible to operate SWIM with different macro-cycles (as mentioned previously) and with a fixed antenna (no rotation).

On-board processing is performed to reduce the data rate: the chirp scaling, the power detection, the swath selection as well as time and range summations are computed. The chirp scaling includes range compression and compensation of range migrations before summation. This migration compensation and the number of integrated samples (or time duration) result from a trade-off between the need for thermal noise and speckle reduction, and the need for keeping a minimum ground horizontal resolution of $35 \mathrm{~m}$.

Finally, the downloaded signal for each cycle consists in the mean backscatter power versus range provided with a range resolution and number of points in the swath as given in Table 3.

Table 3: Range resolution, number of range gates $\mathrm{N}_{\text {range }}$ used for on-board range integration, and number of range bins in the downloaded signal.

\begin{tabular}{|c|c|c|c|c|c|c|}
\hline & $0^{\circ}$ & $2^{\circ}$ & $4^{\circ}$ & $6^{\circ}$ & $8^{\circ}$ & $10^{\circ}$ \\
\hline $\begin{array}{c}\text { Range resolution in } \\
\text { radar geometry (m) }\end{array}$ & 0.47 & 1.88 & 1.88 & 0.94 & 1.41 & 1.41 \\
\hline $\mathrm{N}_{\text {range }}$ & 1 & 4 & 4 & 2 & 3 & 3 \\
\hline $\begin{array}{c}\text { Number of downloaded } \\
\text { range bins }\end{array}$ & 512 & 1026 & 1458 & 2772 & 2784 & 3216 \\
\hline
\end{tabular}

\section{OCEAN WAVE MEASUREMENT PRINCIPLE WITH SWIM}

SWIM is designed to measure the 2D wave spectrum, i.e. the density spectrum of wave heights or wave slopes as a function of the $2 \mathrm{D}$ wavenumber vector $\mathrm{k}$ (expressed here in polar coordinates $(\mathrm{k}, \phi)$ ). We first recall that the wave height spectrum $\mathrm{F}(\mathrm{k}, \phi)$ is defined as the Fourier Transform of the instantaneous spatial autocorrelation of the surface displacements. In polar coordinates the wave slope spectrum $\mathrm{E}(\mathrm{k}, \phi)$ is related to the wave height spectrum $\mathrm{F}(\mathrm{k}, \phi) b y$ :

$\mathrm{E}(\mathrm{k}, \phi)=\mathrm{k}^{2} \mathrm{~F}(\mathrm{k}, \phi)$

Eq.1

The total energy of the waves is usually characterized by the significant wave height Hs by:

$H_{s}=4 \sqrt{\int_{k} \int_{\phi} F(k, \phi) k d k d \phi}$

In the 1990's an alternative concept to SAR systems was proposed by Jackson to measure directional ocean wave spectra both from airborne and spaceborne configurations [11, 2]. It was implemented and validated on various airborne systems such as the Ku-band Radar Ocean Wave Spectrometer (ROWS) developed by Jackson et al. [6,12], the C-band system RESSAC [4], the C-band polarimetric system STORM [13], and more recently the Ku-band KuROS radar [5]. Although a preliminary design for a spaceborne instrument was established in 2000 [3], SWIM will be the first spaceborne instrument based on this principle.

The instrument principle uses the fact that at low incidence angle (around $8^{\circ}-10^{\circ}$ ), the normalized radar cross-section is sensitive to the local slopes related to the tilt of the long waves but almost insensitive to small scale roughness due to wind as well as to hydrodynamic modulations due to interaction of short waves and long waves [11].

For each azimuth direction $\phi$ of the antenna, positions on the mean sea surface may be defined by their horizontal local coordinates $\mathrm{x}$ and $\mathrm{y}$, where $\mathrm{x}$ is horizontal distance along the antenna pointing direction, and $\mathrm{y}$ is along the azimuth direction. The elementary backscatter cross-section $\sigma$ is given by $\sigma=\sigma_{0} A$, where $A$ is the area contained within a radar range gate. The presence of the large sea waves produces a tilt modulation of $\sigma$ given by:

$$
\delta \sigma(x, y)=\sigma(x, y)-\bar{\sigma}(x, y) \quad \text { Eq. } 3
$$


where $\bar{\sigma}(x, y)$ is the mean surface radar cross-section which would occur if no large-scale wave were present. This cross-section is only dependent to small scale roughness with limited impact at this incidence range.

As shown in [11], the fractional variation of the normalized radar cross-section along the direction of propagation of the waves writes:

$\frac{\delta \sigma}{\sigma} \cong\left(\cot \theta-\frac{\partial \ln \sigma_{o}}{\partial \theta}\right) \frac{\partial \zeta}{\partial x}$

Using a real-aperture radar, the fractional modulation $\mathrm{m}(\mathrm{r}, \phi)$ of the cross-section seen by the radar is averaged laterally across the beam and the sea wave polar-symmetric height spectrum $\mathrm{F}(\mathrm{k}, \phi)$ is then obtained from the density spectrum of modulation $\mathrm{P}_{\mathrm{m}}(\mathrm{k}, \phi)$-see [2]:

$P_{m}(k, \phi)=\langle\alpha\rangle k^{2} F(k, \phi)$

Eq. 5

where $\langle\alpha\rangle$ is the value estimated at the center of the beam of the following coefficient:

$\alpha=\frac{\sqrt{2 \pi}}{L_{y}}\left(\cot \theta-\frac{\partial \ln \sigma_{o}}{\partial \theta}\right)^{2}$

where Ly is the $3 \mathrm{~dB}$ azimuthal width of the beam footprint.

To obtain Eq. $(5,6)$, it is assumed that the $\mathrm{L}_{\mathrm{y}}$ dimension is much larger than the wavelength to be detected, which will obviously be satisfied in a satellite configuration $\left(\mathrm{L}_{\mathrm{y}}\right.$ is of the order of $18 \mathrm{~km}$ ). The function $\langle\alpha\rangle \mathrm{k}^{2}$ is called the Tilt Modulation Transfer Function.

Assuming that the signal fluctuations due to speckle and thermal noise can be neglected, the density spectrum $\mathrm{P}_{\mathrm{m}}(\mathrm{k}, \phi)$ is obtained from the measurements as:

$$
P_{m}(k, \phi)=\frac{1}{2 \pi} \int\langle m(x, \phi) m(x+\xi, \phi)\rangle e^{-i k \xi} d \xi
$$

where $\mathrm{m}(\mathrm{x}, \phi)$ is for each radar azimuth direction $\phi$, the signal modulation $\mathrm{m}(\mathrm{r}, \phi)$ projected on the surface, and the angle bracket denotes ensemble average.

In reality, the signal is affected by speckle and thermal noises which are additive in the Fourier domain. In order to correctly retrieve the wave spectra from the signal fluctuations, the impact of these noises must be minimized. For the thermal noise, this is achieved by specifying an appropriate Signal to Noise Ratio (SNR). For the speckle this is first achieved by using a large bandwidth for the transmitted pulse. However speckle reduction by averaging techniques remains limited because high range and temporal resolutions are necessary to retrieve the wave spectra from Eq. (5) to (7). Therefore the speckle must be subtracted in the spectral domain where the signal fluctuation spectrum can be expressed as [2]:

$P_{\delta \sigma_{0}}(k, \phi)=\delta(k)+R(k) P_{m}(k, \phi)+\frac{1}{N_{\text {ind }}} P_{s p}(k)+\frac{1}{N_{\text {ind }}} P_{\text {th }}(k) \quad$ Eq. 8

where $\mathrm{P}_{\delta \sigma 0}$ is the density spectrum of the signal fluctuations, $\mathrm{P}_{\mathrm{sp}}$ and $\mathrm{P}_{\mathrm{th}}$ are the density spectrum of the signal fluctuations due to speckle spectrum, and thermal noise respectively, $\delta(\mathrm{k})$ the Dirac function, $\mathrm{R}(\mathrm{k})$ the density spectrum of the impulse response and $\mathrm{N}_{\text {ind }}$ is the number of independent samples used in the signal estimation (integration in time and range). Assuming a Gaussian shape for the transmitted pulse, Jackson et al [11] showed that the spectrum of the speckle can be expressed as:

$P_{s p}=\frac{1}{\sqrt{2 \pi} N_{\text {ind }} K_{p}} \mathrm{e}^{-\frac{k^{2}}{2 K_{p}^{2}}} \quad$ Eq. 9a

where $\mathrm{K}_{\mathrm{p}}$ is related to the intrinsic resolution of the radar dr, projected on the surface:

$K_{p}=\frac{2 \sqrt{\ln 2} \sin \theta}{d r}$

Eq.9b 
One of the challenges for estimating unbiased wave energy from the signal modulations is the impact of speckle noise whose energy is inversely proportional to the number of independent samples contained in the radar signal. This number of independent samples depends both on radar characteristics (Doppler bandwidth) and on coherence time of scatters. Although uncertainty in $\mathrm{N}_{\text {ind }}$ does not affect the spreading of energy in wavenumber and direction, it may impact the estimation of density spectrum energy. This problem arises both when estimating wave spectra from SAR observations or from real-aperture SWIM-type observations. However in the SWIM case one additional problem arises because the Doppler bandwidth varies with the azimuthal position of the antenna and tends towards zero when the antenna is pointing along the flight track. In addition, the coherence time of scatters over the sea is not well known and probably depends on geophysical conditions (wind, waves). Therefore, a special attention is put in the SWIM data processing to speckle noise estimation and correction (see below).

\section{SWIM PRODUCTS}

Two mission centers will process the SWIM and SCAT data, one in France and one in China. This paper focuses on the French mission center only. The SWIM data will be processed in two-ways:

near real-time processing to provide near real-time Level 0 to Level 2 products ( within 3 hours after acquisition) : this will be carried out at the CNES mission center (called CWWIC).

- $\quad$ delayed time processing to provide Level 2 to Level 4 products: this will be carried out at the IFREMER missions center (called IWWOC).

The SWIM real-time products will contain three main kinds of variables (Fig.4): significant wave height and wind speed from the nadir beam, backscattering profiles from the six beams, and directional wave spectra from the $6^{\circ}, 8^{\circ}$ and $10^{\circ}$ beams.

The Level 2 output consists in 2D wave spectra in cells of $70 \mathrm{~km}$ x $90 \mathrm{~km}$ on each side of the satellite track, as well as wave parameters (significant wave height, peak direction, peak wavenumber) corresponding to up to 3 partitions of the full wave spectra. The partitioning method is a watershed method that we have adapted from [14] to the case of noisy data. Two-dimensional wave spectrum will be given for each SWIM spectrum beam at $\left(6^{\circ}, 8^{\circ}\right.$ and $\left.10^{\circ}\right)$ and also as a combined product. The characteristic parameters of the directional wave spectrum of each partition (confidence intervals, direction, wavelength, energy) will also be contained in the products.

From all incidence beams, the mean $\sigma_{0}$ values will also be provided as a function of incidence and azimuth.

In addition, products from the nadir processing will be merged to the L2 products (significant wave height, normalized radar cross-section and wind speed).

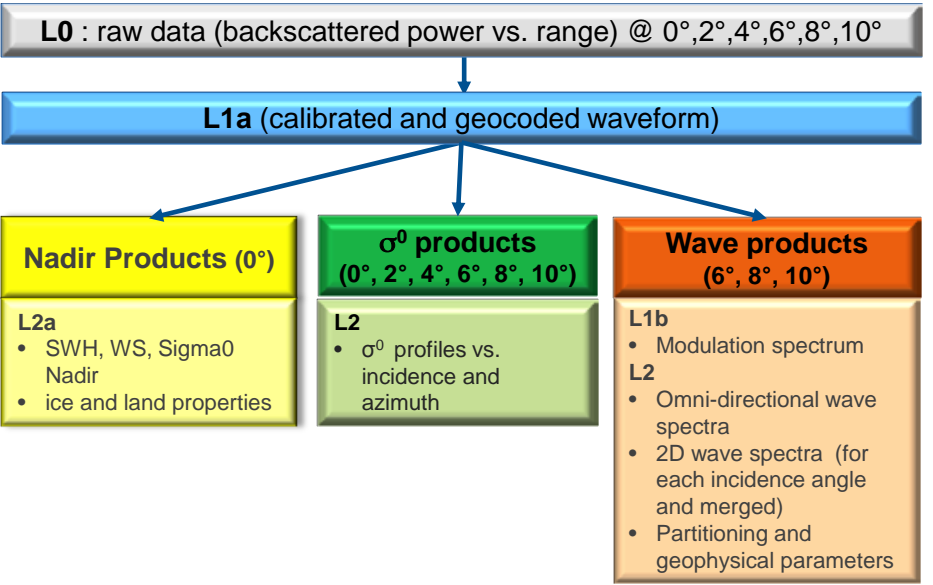

Figure 4. Definition of the SWIM near-real time products 
The SWIM delayed-time products will include level 2S, level 3 and level 4 products :

- the level $2 \mathrm{~S}$ is an alternative level 2 product containing directional wave spectra as well as integrated parameters of the associated wave systems (significant wave height, peak direction and peak wavelength). It will take advantage of the various ancillary data which are in near real-time either not available or of lower quality. Moreover, the wave inversion algorithm will be designed in order to enable wave retrieval also in complex situations such as coastal areas and heterogeneous seas. For example, there will be no attempt to construct 2D wave spectra before partitioning: the wave spectral energy will be partitioned along the SWIM swath. Figure 10 (see section 9 below) illustrates this kind of partitioning, called ribbon partitioning.

- the level 3 is a gridded statistical product based on L2S wave systems. For a given time period and geolocation, it will provide joint statistics from the L2S integrated wave parameters and from the wind given by SCAT. This product will benefit from the angular resolution of SWIM and from the originality of CFOSAT to carry both a wave scatterometer and a wind scatterometer.

- the level 4 is a multi-sensor product providing propagated swell parameters (significant wave height, peak direction and peak wavelength) and its associated storm (location, duration and intensity). Observations from SWIM (L2S product), Sentinel-1 constellation (L2 OCN product) and GF-3 are expected to be the main sources of inputs. These observations are propagated in space and time and are consistently associated to a single storm event (See section 9).

\section{SWIM EXPECTED PERFORMANCE}

\subsection{Geometry and resolution}

The global swath of the instrument is about $90 \mathrm{~km}$ left and right of the nadir track. In the standard mode of operations, the six beams will be successively illuminated, but the azimuth angles sampled during a macro-cycle will not be contiguous (see Fig 2). This is due to mechanical constraints in the implementation of the feed horns on the rotating plate. Nevertheless, the chosen rotation speed of the antenna beams (5.6 rpm) guarantees that for each incidence angle at least two observations (so two cycles) per $15^{\circ}$ azimuth bins are performed over a full rotation. A complete antenna rotation corresponds to a distance of $70 \mathrm{~km}$ along track. The overall sampling of the ground surface after 5 rotations is illustrated in Fig. 5.

The estimation of wave properties with SWIM depends on the surface effective resolution in the elevation direction for the incidence beams $6^{\circ}, 8^{\circ}$, and $10^{\circ}$. This effective resolution results not only from the ground projection of the range resolution after on-board averaging (see Table 3) but also from the compensation of the resolution cell migration during the on-board acquisition time. The chirp scaling can only correct the migration along the range axis (not those due to the curvature of the wave front), and some approximations are made in the chirp scaling algorithm (in particular the Earth rotation effect during the cycle is not corrected). So finally the estimated resolution in the elevation direction is 20 to 35 $\mathrm{m}$ for the incidence beams 6,8 , an $10^{\circ}$. It slightly depends on the position along the orbit, on the incidence beam and on the position within the footprint.

\subsection{Pointing accuracy}

The pointing budget taking into account instrument and platform contributions (with a quadratic summation of all the contributors), leads to:

- $\quad$ pointing elevation accuracy : $<0.25^{\circ}$ (all beams),

- $\quad$ pointing elevation knowledge: $<0.15^{\circ}$ (all beams),

- pointing azimuth knowledge: $<1^{\circ}$ (fixed or rotating antenna). 
These budgets take into account the mechanical mounting precision on the platform, the attitude control accuracy and the mechanical effects on SWIM and the platform (launch, hygrometry, thermo-elastic, etc.).

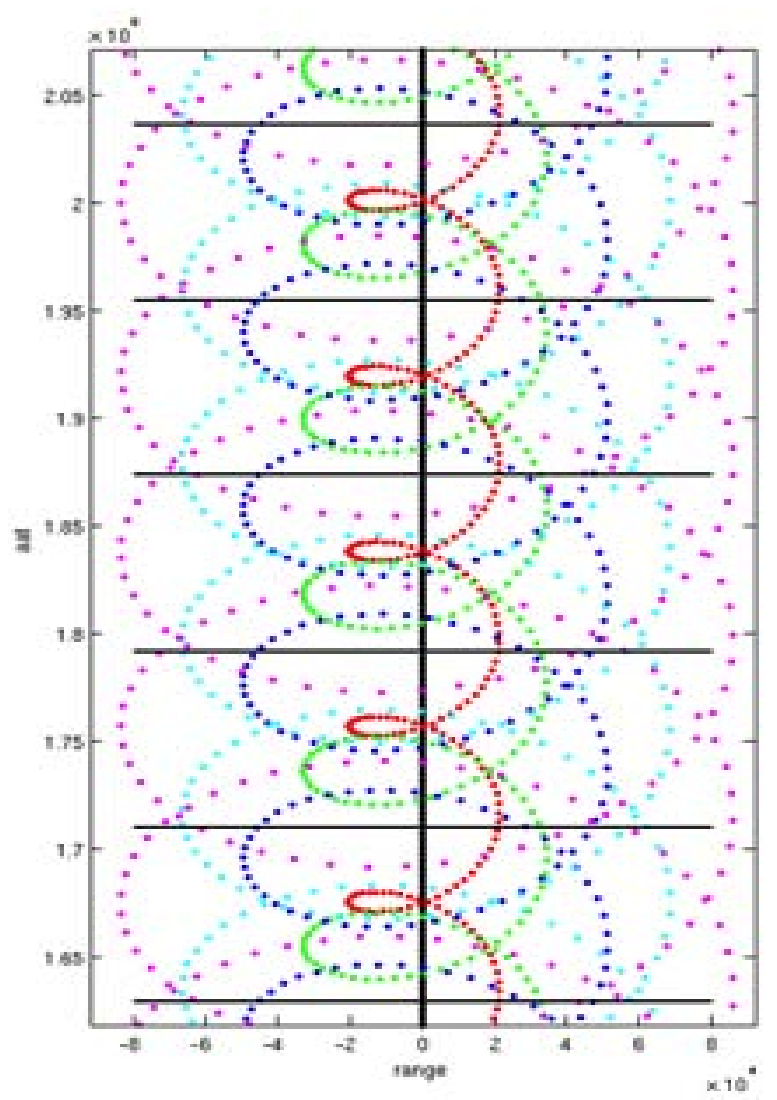

Figure 5: Position of the beam center for each cycle shown as function of the along-track distance (vertical axis in $\mathrm{m}$ ) and acrosstrack distance (horizontal axis in $\mathrm{m})$ for the 6 beams $\left(0^{\circ}\right.$ in black, $2^{\circ}$ in red, $4^{\circ}$ in green, $6^{\circ}$ in blue, $8^{\circ}$ in cyan and $10^{\circ}$ in pink). The horizontal bars delimit the different boxes corresponding to the Level 2 near-real time products

\subsection{Radiometry}

Internal calibration $[15,16]$ is used to reach high precision on the backscattering estimation. Analytical and laboratory estimates of the calibration budgets, taking into account all the inside contributors of the instrument, show that:

- the absolute calibration will be better than $\pm 0.9 \mathrm{~dB}(<0.2 \mathrm{~dB}$ for the random contribution),

- the relative calibration between beams will be better than $\pm 0.2 \mathrm{~dB}$.

Due to the complexity of the SWIM antenna, a very accurate absolute calibration is difficult to reach. Some additional external calibration over surfaces or over cross tracks with other instruments will be necessary in flight.

As for the radiometric accuracy, we estimate that it will be better than $0.26 \mathrm{~dB}$ for data analyzed at the scale of each cycle. Indeed, assuming a normalized radar cross-section of $[8 \mathrm{~dB}, 7.4 \mathrm{~dB}, 6.8 \mathrm{~dB}, 6.2 \mathrm{~dB}, 5.6 \mathrm{~dB}$ and $5 \mathrm{~dB}]$ for the six beams $\left[0^{\circ}, 2^{\circ}, 4^{\circ}, 6^{\circ}, 8^{\circ}, 10^{\circ}\right.$ respectively and an altitude of $545 \mathrm{~km}$, the Signal to Noise Ratio (SNR) at the beam center will be $[27.7 \mathrm{~dB}, 15.2 \mathrm{~dB}, 13.1 \mathrm{~dB}, 10.4 \mathrm{~dB}, 8.1 \mathrm{~dB}, 5.4 \mathrm{~dB}]$ respectively. By taking into account on-board time integration over $\mathrm{N}_{\text {imp }}$ samples (see Table 2) and range-integration over $\mathrm{N}_{\text {ranges }}$ (see Table 3), these values correspond to a minimum radiometric accuracy $\mathrm{K}_{\mathrm{p}}$ of $0.26 \mathrm{~dB}$. For the Level $2 \sigma_{0}$ products, $\mathrm{K}_{\mathrm{p}}$ will be further reduced (better accuracy) by averaging at the scale of $0.5^{\circ}$ incidence step and $15^{\circ}$ azimuth step. 


\subsection{Significant wave height and wind speed from nadir}

The significant wave height will be derived from the waveform as in other altimeter missions. The associated accuracy has been estimated with a simulator similar to those used for recent altimeter missions [17]. It is based on the Brown echo model [18], and a Maximum Likelihood Estimator for the "retracking algorithm" inversion part. These simulations indicate that the accuracy on the significant wave height will be better than $25-30 \mathrm{~cm}$ or $5 \%$ of its mean value. This is fully compliant with the requirement and of same quality as the most recent altimeter performances [19].

The signal-to-noise ratio of the nadir beam signal is high (better than $10 \mathrm{~dB}$ ) and higher by a few $\mathrm{dB}$ compared to that of the most recent altimeter missions. So after in-orbit correction of possible bias on the normalized radar cross-section, standard wind inversion models, similar to e.g. [20,21] should provide wind speed with the same accuracy as the most recent altimeter missions (rms error on wind speed 1.5 to $1.75 \mathrm{~m} / \mathrm{s}$ see [20]).

\subsection{Simulated performance of directional wave products}

\section{a- Methodology}

In order to validate the instrument definition and prepare the processing algorithms, an end-to-end instrument software simulator, SimuSWIM, has been developed. It is an updated and extended version of the simulator previously developed by [3] for the dimensioning of a first version of the system. It includes a direct simulation module (from the surface to the instrument signal) and an inversion module, which includes all the steps that will be used for the inversion of real products when available.

The direct module called SimuSWIM enables to simulate the radar signal from a sea surface topography (twodimensional field of elevation and slopes). This latter is simulated from a pre-defined wave spectrum (empirical formulation or outputs from wave forecast models or in situ measurements). Considering the instrument characteristics and geometry, the beam footprint and range gates are computed on the sea surface. For the six incidence beams, the mean backscattered power is computed assuming a Geometrical Optics backscattering model [22]), and a Gaussian probability density function of wave slopes with mean square slope following the empirical relationship with wind speed of [23]. The signal modulations due to the long waves are then simulated using Eq. (5-6) and the sea surface topography. The simulated radar echoes take into account perturbation by speckle and thermal noises. The on-board processing is also simulated (migrations, on-board averaging over the cycle).

Several simulated data sets have been used to assess the performance of SWIM before launch. In a first stage, two sets of 10min simulated data along the CFOSAT orbit have been used with, as input, wave spectra from hindcasts of the wave model Meteo-France version of the WAM model MF-WAM [24]: a) a case of moderate tropical sea state situation (wind sea and mixed sea conditions) with significant wave height from 1.9 to $2.5 \mathrm{~m}$ and wind speed from 3 to $9 \mathrm{~m} / \mathrm{s} ; \mathrm{b}$ ) a case of high wind and significant wave height in the Indian ocean with significant wave height between 3 and $8 \mathrm{~m}$ and wind speed up to $20 \mathrm{~m} / \mathrm{s}$. In a second stage, we are now considering simulations over one or several complete orbits using as input wave spectra from hindcasts of the ECMWF WAM model.

\section{b- Results}

The L0 data contain for each SWIM beam and each cycle, the output power as a function of range, without radiometric nor geometric corrections (see Fig 6a as an example). From L0 data, the L1a products are calculated by taking into account radiometric and geometric corrections so as to obtain in each footprint, geolocated normalized radar crosssections, which can be referenced as function of the elevation angle (see Fig. 6b). For the radiometric corrections, calibration factors are estimated from the calibration sequences of the instrument. When compared to the TRMM experimental data, Fig. $6 \mathrm{~b}$ shows quite realistic normalized radar cross-section profiles.

The L1b products are intermediate products specific to the SWIM spectrum beams $\left(6^{\circ}, 8^{\circ}, 10^{\circ}\right)$. They will mainly contain, for each azimuth (approximately every $7.5^{\circ}$ in azimuth) and each spectrum beam of SWIM, the density spectra of modulation $\mathrm{P}_{\mathrm{m}}(\mathrm{k}, \phi)$, corrected from impulse response function and speckle density spectra (see Eq. 10).

From the simulations, we could estimate that assuming nominal functioning of the radar, the impulse response has a negligible impact on the products. As for speckle correction, in the nominal processing, the level of speckle will be 
estimated using Eq. [9a]. Two versions of this method will be implemented: i) by fixing the number of independent samples from the radar characteristics ( "Speckle $1 \mathrm{~A}$ "); ii) by using a parametric model relating $\mathrm{N}_{\text {ind }}$ to sea-sate conditions (wind or $\sigma_{0}$, waves,..)- "Speckle $1 \mathrm{~B}$ ".

In addition, alternative methods will be implemented to estimate the energy density of speckle and parameterize its behavior as a function of surface conditions:

- $\quad$ speckle 2: from the noise floor of the fluctuation spectra, using either the noise floor at $\mathrm{k}>0.2 \mathrm{~m}^{-1}$ for each azimuth angle (method 2A), or from the level of the fluctuation spectrum in the azimuth direction where it is minimum (method $2 \mathrm{~B}$ ); these methods assume that no wave signal is detectable at large $\mathrm{k}(2 \mathrm{~A})$ or in some azimuth directions $(2 \mathrm{~B})$;

- Speckle 3: for particular mode of acquisitions (successive acquisitions on the same incidence beam), the crossspectrum method proposed by [25] should provide modulation spectra free of speckle; difference between auto and cross-spectra will provide the density spectrum of speckle;

- Speckle 4: density spectrum of speckle estimated using the specific mode of acquisition named "speckle" mode; the method will be based on the approach presented in [4].

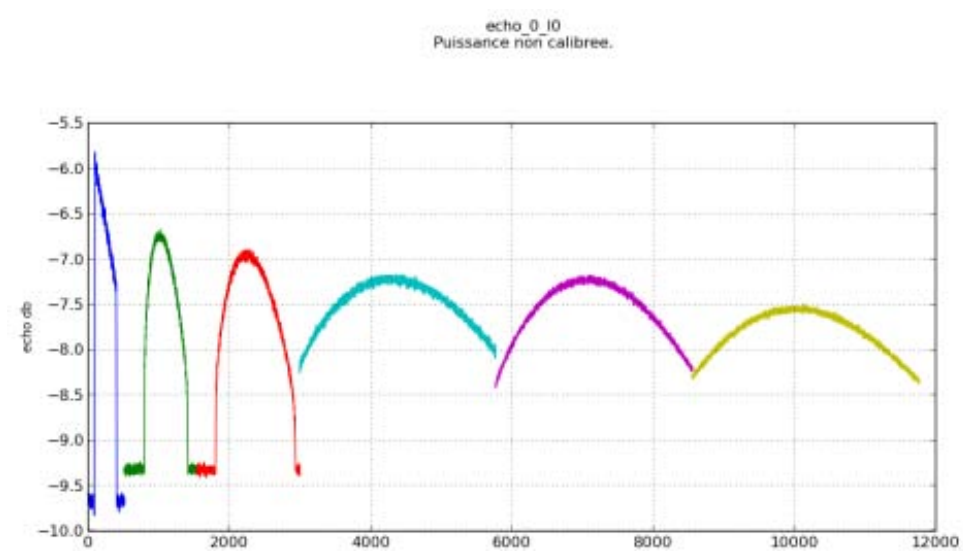

Figure 6a: Example of the L0 output power as a function of range. The results from the six beams are presented on the same plot.

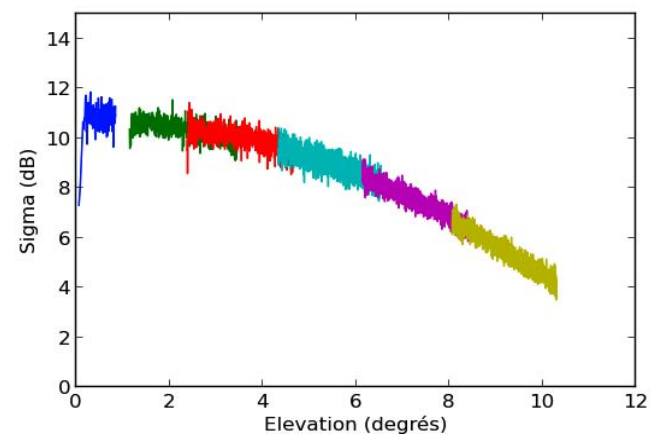

Figure 6b: Example of the L1a output for the normalized radar cross-section plotted here as a function of the elevation angle.

Figure 7 illustrates the density spectrum of modulation and of speckle obtained at the Level1b, from one case of the simulation (set a). It corresponds to an estimation in a given azimuth direction (chosen here along the wave propagation direction). The speckle level estimated from the different methods mentioned here-above is illustrated by curves in color. It shows that although the density spectrum of modulation remains quite noisy, the wave signal can be distinguished. It also shows that the speckle level significantly depends on the chosen method, although it is always close to the pedestal of the spectrum. From this illustration, we expect that the performance on the Level2 products in terms of wave energy will depend on the speckle correction, especially in case of low wave energy.

Because simulations may not be completely representative of the reality (uncertainties on the properties of echo correlation), all the methods presented above to estimate the speckle density spectrum will be used at the beginning of the mission in order to decide which is the most appropriate.

The Level 2 products refer to geophysical data representative of geographic boxes of about 70 x $90 \mathrm{~km}^{2}$ which will contain for each incidence beam and on each side of the track, all azimuth angles within $\left[0-180^{\circ}\right]$. Within these boxes, directional wave spectra will be given for each of the antenna beam $\left(6\right.$ or 8 or $\left.10^{\circ}\right)$ also as a combined product by merging the products of the three antenna beams $\left(6,8\right.$, and $\left.10^{\circ}\right)$. 


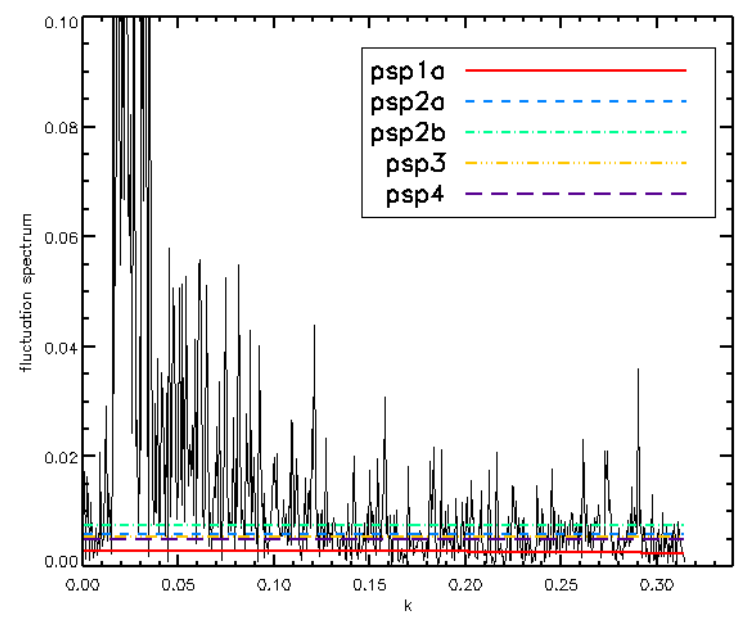

Figure 7: Example of fluctuations spectrum as a function of wavenumber. The mean level of five speckle estimations is overlayed in color. This sample corresponds to a radar echo obtained with the $8^{\circ}$ incidence beam in the wave propagation direction (see text).

In order to convert modulation spectra into wave slope spectra, three options will be implemented for the tilt MTF.

The first one (MTF1) corresponds to Eq.6, where the tilt MTF is estimated from the Level $2 \sigma_{0}$ data (mean profiles estimated in the same geographical boxes as the wave spectrum). This will be the option by default. The second method (MTF2A) uses an empirical model of $\partial \sigma_{0} / \partial \theta$ provided by existing Ku-Band radar data sets (presently from the TRMMPrecipitation radar [26]). Using these data sets, look-up tables relating the tilt MTF to wind speed and significant wave height have been established. With wind speed and significant wave height provided by the nadir beam or by ancillary data from meteorological models, the tilt MTF will be estimated from these look-up tables regularly updated by recent data sets -eg. GPM [27].

The third method (MTF2B) is to assume that the normalized radar cross-section is related to the sea surface slope probability density function (pdf) through the Geometrical Optics backscattering model [22]. Assuming in addition that the slope pdf is an isotropic Gaussian function whose standard deviation (mean square slope-mss) is related to the wind speed, the MTF2B expression becomes:

$<\alpha>=\frac{\sqrt{2 \pi}}{L_{y}}\left(\cot \theta-4 \tan \theta+2 \frac{\tan \theta}{m s s(U)}\right)$

where the wind speed $U$ is given by ancillary data (meteorological model or product from the nadir beam or from the SCAT data) and mss(U) is given by [23] or equivalent updated empirical models.
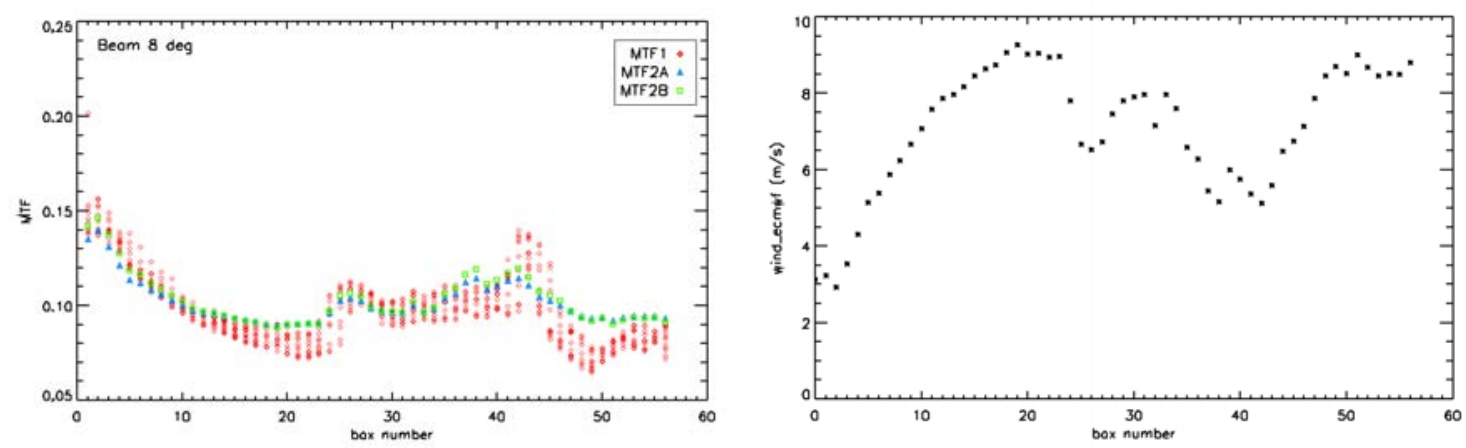

Figure 8: (left) coefficient $\langle\alpha\rangle$ of Eq.(5) for the incidence beam $8^{\circ}$, estimated by using MTF1, 2A, 2B calculation (red and pink: MTF1, blue : MTF 2A, green : MTF2B). The $\langle\alpha\rangle$ coefficient is plotted as a function of the product cell number along the track. (left):

The red and pink symbols (MTF1) are spanned over all the azimuth directions whereas the results for MTF2A and MTF2B are independent of the azimuth angle. (right): corresponding wind speed used in the simulation 
Figure 8 shows that the MTF1, MTF2A and MTF2b are globally consistent. In homogeneous conditions, the azimuthally-dependent MTF estimate (MTF1) provides results very similar to those of the other methods. The choice of the method to be used after launch will be made after CAL/VAL analysis

Figure 9 illustrates a wave slope spectrum provided as output of the Level 2 processing chain compared to the reference (input of the simulation). The reference is the MF-WAM 2D wave slope spectrum used as input of the simulation. This example corresponds to a mixed sea case with a swell and a wind-sea component, both in the same direction as shown in Fig 9a. The total significant wave height is $4.65 \mathrm{~m}$. Fig.9b shows the corresponding 2D spectrum after simulation of SWIM signal and application of the retrieval processing. Qualitatively the agreement is good: consistency of peak and dominant direction (accounting for a $180^{\circ}$ ambiguity in the SWIM result). Three partitions are detected, the first two in close agreement with the reference spectrum .The third partition encompasses mainly noise. The omni-directional wave height spectrum (Fig. 9c) is also in good agreement with the reference, with however a slight overestimation of the wave height energy density close to the peak of the spectrum.
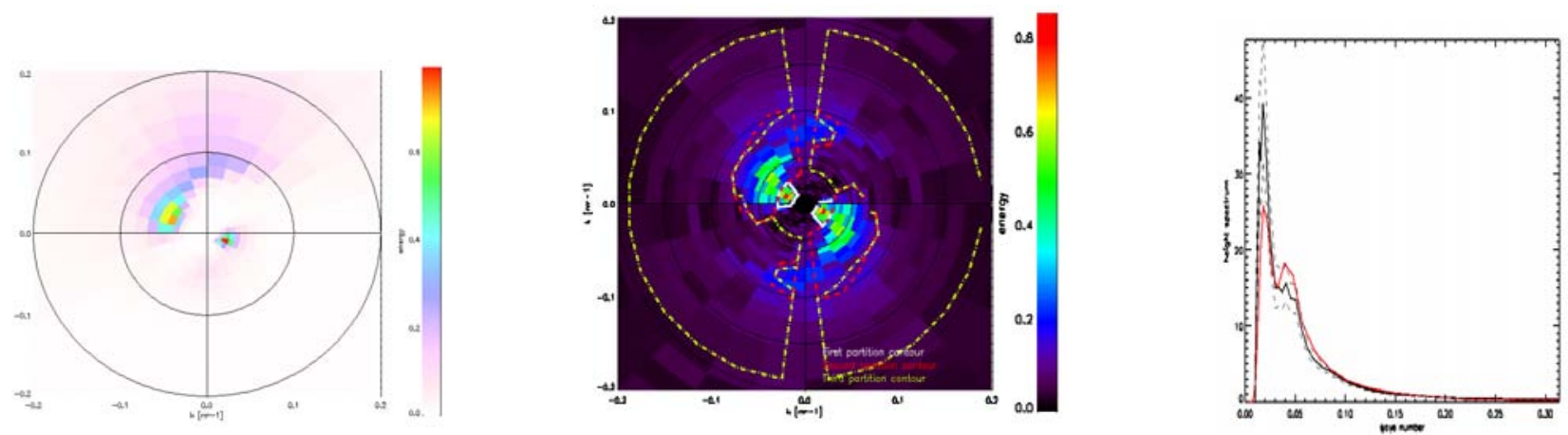

Figure 9: (a) Wave slope 2D spectrum used as input of the simulation. (b) 2D wave slope spectrum as output of the simulation. In (a) and (b), energy in color level, wave number as distance from the center, wave propagation direction as angle from the North- with a $180^{\circ}$ ambiguity in (b). In (b), the detected partitions are indicated as solid white, dashed red and dashed yellow lines.(c) Corresponding omni-directional wave height spectrum : the red solid line is the reference, the black solid line is the result of the inversion. The confidence interval is indicated as dashed lines.

Figure 10 illustrates the wave parameters as obtained from a L2S partitioning (applied on simulated data) : in this case, the partitioning is applied along the original SWIM geometry without using the boxes illustrated in Fig.5.

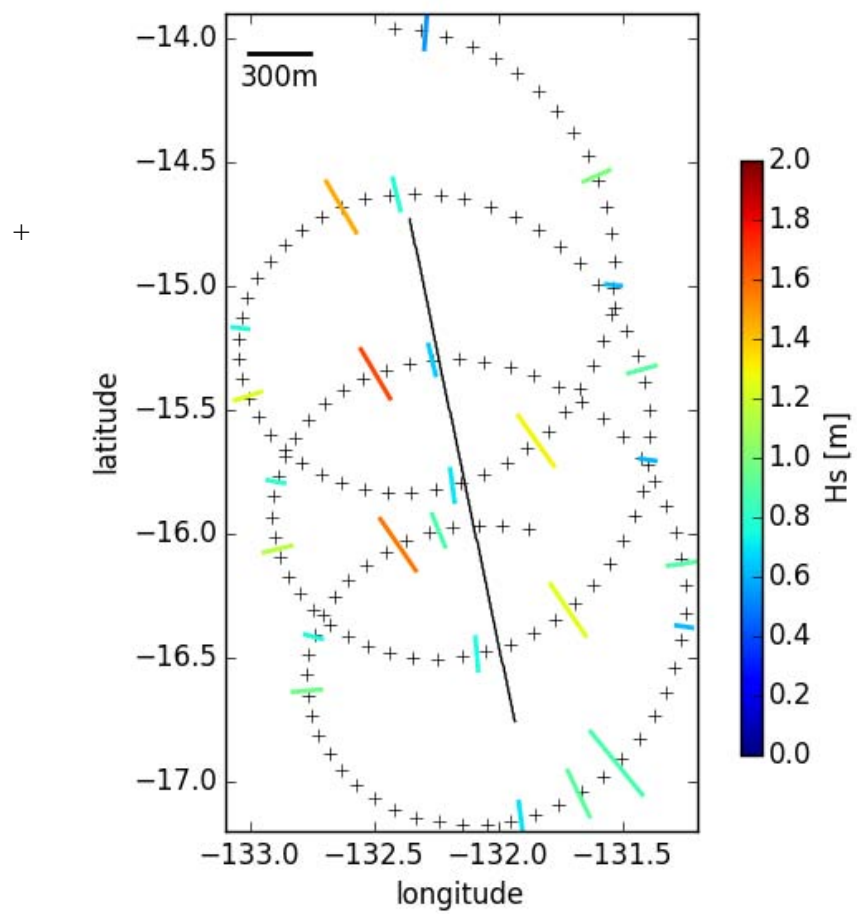

Figure 10: Example of L2S ribbon partitioning for a given incidence. Black straight line indicates the SWIM track, black markers indicate SWIM footprint centers. The partitions are shown with colored sticks whose orientation, length and color depend respectively on the peak direction, peak wavelength and significant wave height. 
In order to quantify on a statistical basis the quality of the inverted products, and to compare the results from the three incidence beams and from the different options for speckle and MTF corrections, we analyze here several statistical parameters namely mean and relative errors on the total energy, peak wavenumber and peak direction for each partition, as well as standard deviations and scatter index. These are calculated with respect to the reference (called ref) which refers to the partition of the reference spectrum. For this performance analysis, the mask of the partition for each reference $2 \mathrm{D}$ spectra is fixed as identical to the one obtained from the corresponding inverted spectrum.

We define for each wave spectrum the mean bias and standard deviation around the mean for the energy, peak wave number and peak direction of each partition. The peak wavenumber is estimated as the energy-weighted mean wavenumber over \pm 5 wave number bins around the peak. The peak direction of each partition is estimated as the energyweighted mean direction over \pm 3 direction bins around the peak.

Figure 11 shows the error statistics on energy (top), peak wave number (middle) and peak direction (bottom) for the first two partitions detected in each inverted spectrum. This analysis is based on the analysis of 110 wave spectra for each incidence beam along the 10 min sub-orbit. Partitions are ranked according to their total energy.
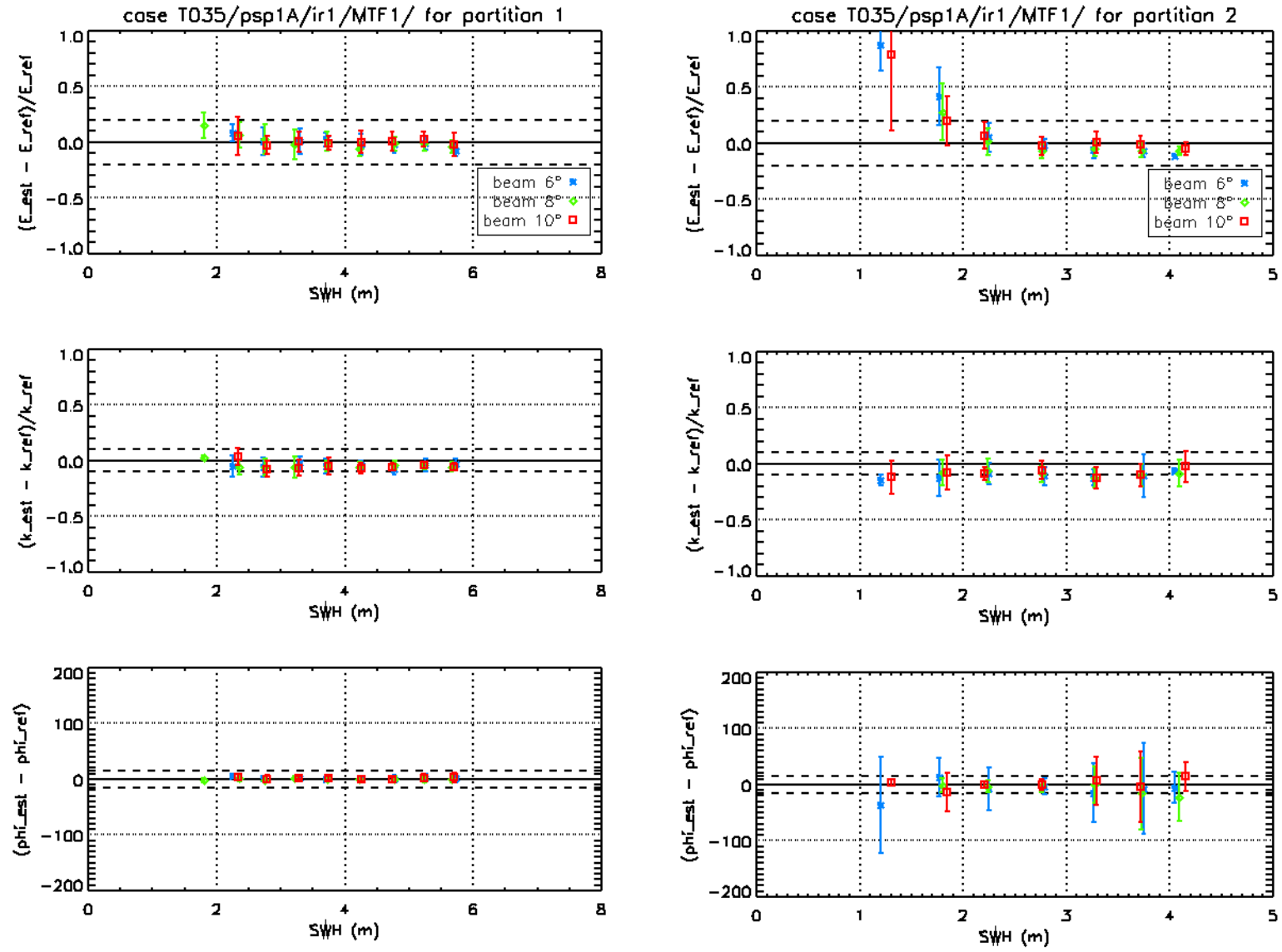

Figure $11: 10$ min sub-orbit statistics of relative errors on wave energy (top) dominant wave number (middle) and dominant direction (bottom) as a function of reference significant wave height for partition 1 (left panel) and 2 (right panel). The symbol colors refer to the SWIM incidence beam (see inserted legend)

Figure 11 illustrates for the test case (b) (10 min portion of orbit over the Indian Ocean) that the parameters of the first partition of the wave spectra are retrieved with an accuracy which is fully compliant with the specification: bias and standard deviation are less than $20 \%$ in energy, $10 \%$ in wavenumber and $15^{\circ}$ in direction. Results for the second partition show higher errors, with significant bias in energy for partitions with wave height less than $2 \mathrm{~m}$. The second partition 
may be affected by a less precise partitioning and by extra noise coming from the principal partition. The results for the third partition (not shown) are of lesser quality. This is likely because the third partition encompasses a large portion of noise in the spectrum. Same conclusions are reached from the analysis of the full orbit test and from the test case (a) Pacific ocean. In summary these tests show that:

- the bias and standard deviations in energy, direction and peak wavenumber remain within the initial specification, provided the significant wave height is larger than $1.5 \mathrm{~m}$ for the first partition and $2 \mathrm{~m}$ for the second partition.

- performance on the third partition is rather poor and shows that the partitioning algorithm should be improved regarding the detection of this $3^{\text {rd }}$ partition.

- $\quad$ the performances are almost the same for the three SWIM incidence beams $\left(6,8,10^{\circ}\right)$

\section{TIME-SPACE ANALYSIS OF WAVE FIELDS FROM SATELLITE DATA}

Since April 2014, Sentinel-1 A is routinely measuring 2-D ocean swell spectra over open-ocean. Except in the north east Atlantic region, the coverage is global. Sentinel-1 B will have the same capacities. Its launched is announced for April 2016. The common Chinese/French CFOSAT satellite will be launched in 2018. Then, GF-3, a high resolution C-band SAR should be launched in 2019 by the Chinese Agency CNSA. In the line of ENVISAT/ASAR, GF-3 will carry onboard a SAR with a Wave Mode to measure 2-D ocean swell spectra over open-ocean at global scale. Given the expected life duration of these missions, we should be in position of getting 2-D ocean wave measurements (level-2 products) from 4 different satellites in 2020. This would be a world premiere.

As for other geophysical variables such as ocean surface wind or sea surface temperature, we will take benefit of the diversity of these existing wave measurements to provide an homogeneous and qualified dataset at the highest resolution possible in time and space. A product, which will merge data from these different missions is defined as a level-4 product. To date, such a product from ocean waves spectra measurements does not exist.

However, to be properly merged, the measurements have to be consistent and the limitations of each sensor well understood and taken into account. As a consequence, the validation of each mission and the inter-calibration between the different sensors represents two mandatory steps prior any data combination.

As defined fro the mission center IWWOC, waves L4 product aims at gathering together all the wave measurements originating from the same storm. It will provide the main integrated parameters (wavelength, direction, period and significant wave height) of ocean waves and of its associated storm (location, duration, intensity).

The concept has already been applied to ENVISAT/ASAR data [28,29]. We recall the main steps to make such a product below:

1. Observations collection and filtering: Wave systems parameters are estimated from 2-D spectra coming from various sources. Observations are filtered with respect to their quality.

2. Propagation: Observations and its associated integrated parameters are then propagated in space and time, backward and forward. Propagation is done according to the wave direction given by the observation at its group velocity $\mathrm{c}(c=1 / 2 \sqrt{ }(g \lambda / 2 \pi))$ where $\lambda$ is the wavelength of the wave partition to propagate $g$ the acceleration of gravity constant. We assume deep ocean and no wave-current interactions during the propagation. Wind-wave interactions are also neglected. As a consequence if at a certain time and location the wind speed is too high with respect to the phase speed of the wave propagated numerically, then the propagation is stopped.

3. Refocusing: Back-propagated swell observations are analyzed to determine the storm source location in both time and space. For the storm source description, the back-propagated wave analysis is also complemented by wind measurements analysis.

4. Significant wave height: As already mentioned, outside island shadows and in the absence of ocean currents, swell propagates at group velocity and along geodesics in deep water. Under this assumption, the only 
phenomena modifying the swell energy during the propagation are (i) angular spreading, acting in transverse direction, and frequency dispersion, acting in radial direction and (ii) dissipation. As proposed in [28,29], the swell dissipation far from the storm source $(\mathrm{d}>4000 \mathrm{~km})$ can be parameterized by:

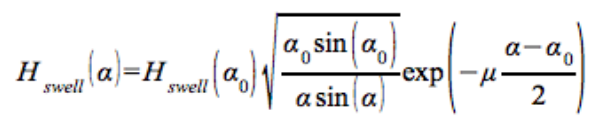

where $\alpha$ is any angular great-circle distance from the storm source and $\alpha_{0}$ a angular great-circle distance from the storm source associated to an observation where the significant wave height of the swell has been measured; $\mu$ is a constant.

5. Consistency of data at storm scale: Observations that could not be associated to a storm are filtered out. For a given storm, the consistency of associated wave observations with respect to significant wave height distribution is checked. Outliers are filtered out.

Swell parameters associated to qualified observations and propagations are the main components of the product (see examples in [28]). The time sampling of the product is expected to be regular (at least $6 \mathrm{~h}$ ). The space sampling is not regular and fully driven by observations and propagated observations. However, the data that have been filtered out can contain valuable information. For instance in the particular case of CFOSAT/SWIM, as the minimum detected wavelength will be smaller than with other sensors (up to $70 \mathrm{~m}$ ) we may reject waves that has been affected by current during their propagation. Wave-current interactions are still a subject for research. As a consequence, the Level-4 method proposed here could also serve to map the area where the waves propagation is affected by current.

\section{ASSIMILATION OF WAVE SPECTRAL INFORMATION IN WAVE FORECASTING MODELS}

In the framework of CFOSAT satellite mission, Météo-France has implemented an assimilation system specifically for the use of wave spectral information. This system is using a partitioning principle to split the wave spectrum in different wave trains, and then computing mean parameters of every partition. When the observed partition is assigned to first guess partition from the wave model, then an optimal interpolation is performed on the mean parameters. Afterward the analyzed partitions are superposed to build analyzed wave spectrum [30]. The assimilation system has been used operationally with the SAR wave spectra from Envisat since 2011 and until the end of mission on March 2012. Major lesson from this experience is that the impact of the assimilation of wave spectra induced $20 \%$ of improvement on root mean square errors of the peak period of long waves exceeding $200 \mathrm{~m}$ of wavelength [31].

The operational wave forecasting system of Météo-France has been upgraded in November 2014 with major improvements thanks to the My wave European FP7 research project [32]. The system is composed of the wave model MFWAM and the assimilation system using the wave observations from satellites. The model MFWAM is based on the ECWAM code with a dissipation by wave breaking and a swell damping term induced by the air friction at the sea surface developed by Ardhuin et al. [33]. The assimilation system is based on an optimal interpolation technique for significant wave heights from altimeters and for spectral information, assimilation of main wave parameters (wave height, mean period and direction) of up to 3 partitions of the full two-dimensional spectrum.

In order to prepare the use of wave spectra from CFOSAT, wave model runs with assimilation of SAR and synthetic wave spectra have been performed during these last years in order to evaluate the impact of using such data on the wave forecast. To perform OSSE (Observed System Simulation Experiment) experiments, synthetic wave spectra are obtained from the run of the wave model driven by analysed winds. A random function has been applied on the synthetic spectra in order to avoid correlation between the observations and the first guess. The second method is based on using the data simulator FAWASSI developed by the french space agency CNES. This simulator uses as input an ocean surface reconstructed from directional wave spectra provided by the wave model MFWAM. It is a simplified version of the simulator describe in section 8.5.a here-above. As an output the simulator FAWASSI provides synthetic spectra as they are seen by the instrument SWIM of CFOSAT. The wave model runs with assimilation of synthetic wave spectra is driven by forecasted winds in order to decorrelate the synthetic observation and the first guess from the model. 
Figure 12 shows an example of an omni-directional wave spectrum before and after the assimilation of SAR wave spectrum in the wave model MFWAM during the hurricane Katia in September 2011. In this case the wave model underestimated the peak energy and it is clear to see that after the assimilation the model matches up the SAR wave spectrum.

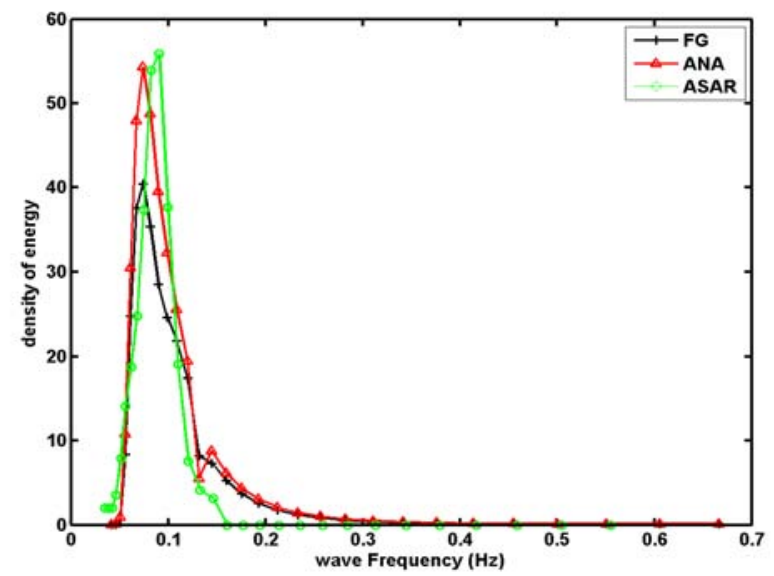

Figure 12: Omni directional wave spectrum as a function of wave frequency. Black, red and green lines give the spectrum before assimilation (first guess from the wave model MFWAM), after the assimilation and the SAR wave spectrum, respectively.

Figure 13 indicates the impact of the assimilation of CFOSAT wave spectra on mean wave period after 1-day forecast. This reveals that the impact remains significant with a mean wave period correction due to assimilation of up to about 2 seconds in all the ocean basins. In other respects by comparison with altimeters wave heights it has been demonstrated that the persistency of the assimilation of CFOSAT wave products stays efficient until roughly 3 days of forecast as illustrated in figure 14 .

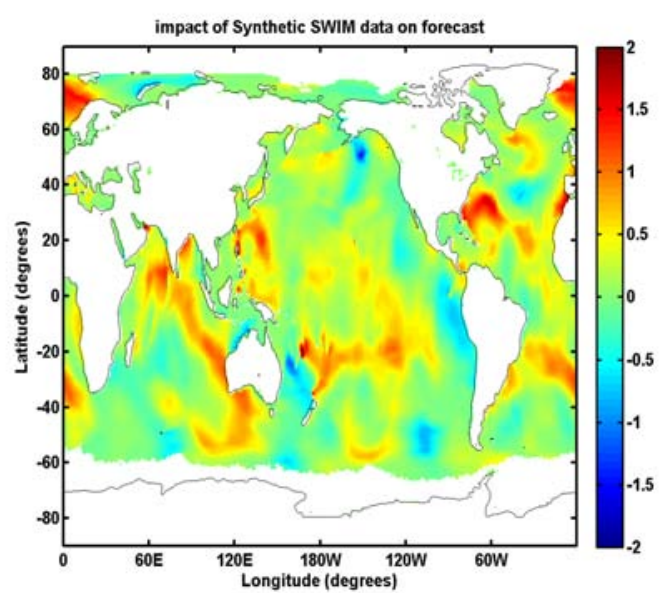

Figure 13: Difference between mean wave period from the model MFWAM with and without assimilation of CFOSAT wave data (significant wave height and synthetic wave spectra) on September $26^{\text {th }} 2011$ at 0:00 (UTC). The positive and negative values indicate the underestimation and overestimation of the wave model before the assimilation.

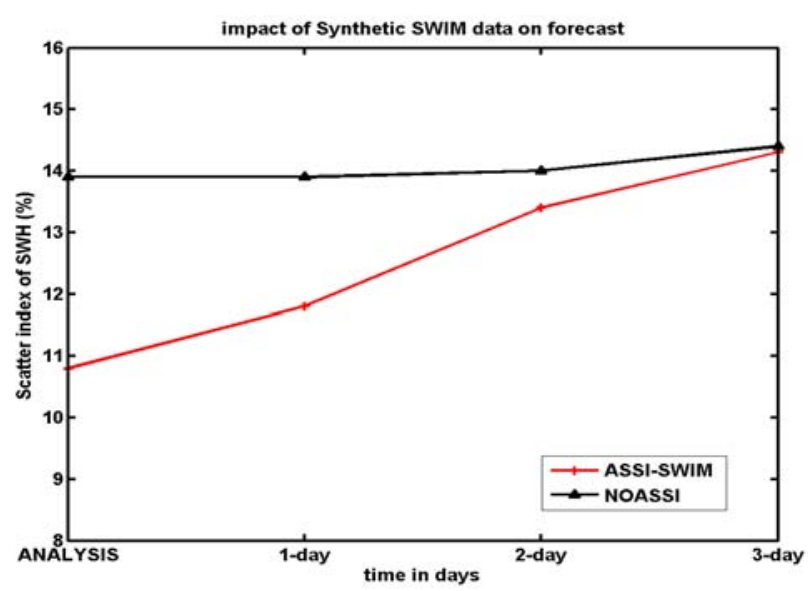

Figure 14: The variation of normalized scatter index of significant wave height in the period of forecast. Comparison performed with altimeters Jason-1 and Jason-2 during September 2011. Red and black colors stand for runs with and without assimilation of CFOSAT wave products. 


\section{1- CONCLUSION}

CFOSAT is a very innovative and promising mission, which will provide new observations of wave and wind fields from a dual radar system in Ku-Band (the wave scatterometer SWIM and the wind scatterometer SCAT). The first data should be delivered to the interested community in 2018. Work is presently under progress to prepare the future use of these data: finalization of the processing chains and their implementation in mission centers, preparation of the CAL/VAL operations including in situ and airborne field campaigns, preparation of high-level products to combine different kinds of wave and wind satellite data, assimilation of data in numerical models.

\section{REFERENCES}

[1] Lin W., and X. Dong, "Design and optimization of a Ku-band rotating, range-gated fanbeam scatterometer", Int. Jour. Remote Sensing, (2011)

[2] Jackson F. C., T.W. Walton, and P.L. Baker, "Aircraft and satellite measurement of ocean wave directional spectra using scanningbeam microwave radars", Journal of geophysical research, Vol. 90, No. C1, 987-1004 (1985)

[3] Hauser D., E. Soussi, E.,Thouvenot, L. Rey, "SWIMSAT: A real aperture radar to measure directional spectra of ocean waves from space, Main characteristics and performance simulation", Jour. Atmos. and Oceanic Tech, vol 18 No3, $421-437$ (2001)

[4] Hauser, D., G. Caudal, G.J. Rijckenberg, D. Vidal-Madjar, G. Laurent, and P. Lancelin, RESSAC: A new airborne FM/CW radar ocean wave spectrometer", IEEE Trans. Geosci. Remote Sensing 30 (5), 981-995 (1992)

[5] Caudal G., D. Hauser, R. Valentin, C. Le Gac, KUROS "“A new airborne Ku-band Doppler radar for observation of surfaces”, Jour. Atmos. and Oceanic Technology, Vol. 31, No. 10. , 2223-2245 (2014)

[6] Hasselmann K., and S. Hasselmann, "On the nonlinear mapping of an ocean wave spectrum into a synthetic aperture radar image spectrum and its inversion”, J. Geophys. Res Oceans, Volume 96, C6, 10713-10729 (1991)

[7] HaidenT., M. Janousek, P. Bauer, J. Bidlot, M. Dahoui, L. Ferranti, F. Prates, D.S. Richardson and F. Vitart, "Evaluation of ECMWF forecasts, including 2014-2015 upgrades", ECLMWF technical memorendum 765 (2015)

[8] Ardhuin F., B. Chapron, and F. Collard, "Observation of swell dissipation across oceans", Geophys. Res. Lett., 36, L06607, doi:10.1029/2008GL037030 (2009).

[9] Breivik O, K. Mogensen, J-R Bidlot, M. Alonso Balmaseda, P. A.E.M. Janssen, "Surface Wave Effects in the NEMO Ocean Model: Forced and Coupled Experiments", J. Geophys. Research, Doi: 10.1002/2014JC010565, (2015)

[10] Hemer, M.A., X.L. Wang, J.A. Church and V.R. Swail, "Coordinated global ocean wave projections”, Bull. Amer. Meteor. Soc., 91(4), 451-454. DOI: 10.1175/2009BAMS2951.1 (2010)

[11] Jackson, F. C., An analysis of short pulse and dual frequency radar techniques for measuring ocean wave spectra from satellites, Radio Sci., 16, 1385-1400 (1981).

[12] Jackson F. C., W. T. Walton, and C. Y. Peng, "A comparison of in situ and airborne radar observations of ocean wave directionality”, J. Geophys. Res., Vol. 90, 1005-1018, (1985)

[13] Hauser D., T. Podvin, M. Dechambre, R. Valentin, G. Caudal, J.-F. Daloze, "STORM : A new airborne polarimetric real-aperture radar for Earth observations", in Proceedings of POLINSAR Workshop (Workshop on Applications of SAR Polarimetry and Polarimetric Interferometry), ESA SP-529, Frascati, Italy, 14-16 january 2003, (2003)

[14] Hanson J.L.H., Phillips O., “Automated Analysis of Ocean Surface Directional Wave Spectra”, J.A.O.T., vol. 18, p.277 (2000)

[15] Enjolras V., L. Rey, T. Amiot, C. Tison, P. Castillan, "SWIM, a state of the art multi-incidence beams Ku-band waves scatterometer to go beyond current radar systems", in IGARSS'09, July 2009, (2009)

[16] Lorenzo, J., F. Demeestere, J. Brossier, S. Pouyez, V. Enjolras, L. Rey, T. Amiot, C. Tison, P. Castillan, "Next generation of multi beam rotating antenna on SWIM scatterometer", in IGARSS'10, Page(s): 3478 - 3481, July 2010, (2010)

[17] Thibaut P., N. Steunou, O.Z. Zanife, G. Carayon, E. Thouvenot, "The Poseidon-2 altimeter simulator of performances", in IGARSS'01, 7, 3212-3214, July 2001, (2001)

[18] Brown G.S., "The average impulse response of a rough surface and its applications", IEEE Trans. Antennas Propag., 25, 67-74, (1977)

[19] Abdalla S., P.A.E.M. Janssen, J.R. Bidlot, "Jason-2 OGDR Wind and Wave Products: Monitoring, Validation and Assimilation, Marine Geodesy", 33, 239-25, (2010)

[20] Gourrion J. , D. Vandemark , S. Bailey , B. Chapron , G. P. Gommenginger , P. G. Challenor , M. A. Srokosz ,“A Two-Parameter Wind Speed Algorithm for Ku-Band Altimeters", Journal of Atmospheric and Oceanic Technology, Vol. 19, No. 12, (2002)

[21] Witter D.L. and D.B. Chelton, "A GEOSAT wind speed algorithm and a method for altimeter wind speed algorithm development”, J. Geophys. Res., 96, 8853-8860, (1991) 
[22] Valenzuela, G.R., "Theories for the interaction of electromagnetic and ocean waves- A review", Boundary Layer Meteorol., 13, 61-85 (1978)

[23] Freilich M. H., and B. A. Vanhoff, "The relationship between winds, surface roughness, and radar backscatter at low incidence angles from TRMM Precipitation Radar measurements", Journal of Atmospheric and Oceanic Technology, Vol. 20, 549-562, (2003)

[24] Lefèvre J.M., Aouf L., Bataille C., Ardhuin F., Queffeulou, P. . Apport d'un nouveau modèle de vagues de $3^{\circ}$ génération à Météo France. Actes de conférence des Ateliers de Modélisation de l'Atmosphère, 27-29 janvier 2009, Toulouse, France, (2009).

[25] Engen, G., and H. Johnsen, "SAR-Ocean wave inversion using image cross spectra", IEEE Trans. Geosci. Rem. Sens., Vol. 33, No. 4, (1995).

[26] Tran N., B. Chapron, D. Vandemark, "Effect of long waves on Ku-Band ocean radar backscatter at low incidence angles using TRMM and altimeter data", IEEE Geoscience and Remote Sensing Letters, 4 (4), doi: 10.1109/LGRS.2007.896329 (2007)

[27] Nouguier F., A. Mouche, N. Rascle, B. Chapron and D. Vandemark, "Analysis of dual-frequency ocean backscatter measurements at $\mathrm{Ku}$ - and Ka-band using near-nadir incidence GPM radar data », GRSL,. submitted, in révision

[28] Collard F. F. Ardhuin and B. Chapron, « Monitoring and analysis of ocean swell fields from space: New methods for routine observations », J. Geophys. Res. vol 114, C07023, doi:10.1029/2008JC005215, (2009)

[29] Ardhuin F. Chapron B., and F. Collard, « Observation of swell dissipation across oceans, Geopgys. Res. Letters », Vol. 36, L06607, doi:10.1029/2008GL037030, (2009)

[30] Aouf L., J-M. Lefèvre, D. Hauser, Assimilation of directional wave spectra in the wave model WAM : an impact study from synthetic observations in preparation to the SWIMSAT satellite mission. Journal of Atmospheric and Oceanic Technology. Vol. 23, No. 3, pages 448-463, (2006).

[31] Aouf L., J-M. Lefèvre, "On the assimilation of ASAR directional wave spectra in the operational wave model MFWAM", Proceedings of workshop SEASAR 2012, Tromso, Norway, (2012).

[32] Janssen P., O. Breivik, L. Aouf, L. Cavaleri, K. Christiensen, G. Korres, final report of work package I of My wave project, 2014. [33] Ardhuin F., R. Magne, J-F. Filipot, A. Van der Westhyusen, A. Roland, P. Quefeulou, J. M. Lefèvre, L. Aouf, A. Babanin and F. Collard : Semi empirical dissipation source functions for wind-wave models : Part I, definition and calibration and validation at global scales. Journal of Physical Oceanography, 40(9), pages 1917-1941, (2010) 